\title{
Motor imagery in conversion paralysis
}

\author{
Karin Roelofs, Gérard W.B. Näring, Ger P.J. Keijsers, and \\ Cees A.L. Hoogduin
}

University of Nijmegen, The Netherlands

Gerard P. van Galen

Nijmegen Institute for Cognition and Information, The Netherlands

Eric Maris

University of Nijmegen, The Netherlands

\begin{abstract}
Introduction: Motor imagery was implicitly and explicitly cued in patients with conversion paralysis. Patients' explicit motor imagery of affected limbs was expected to be slowed. Methods: Six patients and six healthy controls performed an implicit mental motor rotation task (a hand and foot judgement task), an explicit mental motor rotation task, and two control reaction time (RT) tasks. Results: On the implicit task patients showed a significantly larger increase in RT per degree of rotation (RTR) than controls. There was a nonsignificant trend showing a larger RTR for the most severely affected limb in patients. This effect was stronger for the explicit hand rotation task, in which patients' RTR was larger for the most severely affected arm. Furthermore, in $9 \%$ of the explicit hand rotation trials and in $51 \%$ of the explicit foot rotation trials patients showed a complete inability to form a mental image of their most severely affected limb. Patients were also slower on two control RT tasks. Conclusion: General slowing most explicitly manifested itself in tasks that chiefly draw on intentional operations (i.e., the explicit mental rotation task) and is strongest in the most severely affected limb. The findings suggest a general slowing of motor initiation, due to impaired functioning of explicit, intentional processes in patients with conversion paralysis.
\end{abstract}

Correspondence should be addressed to Karin Roelofs, Department of Clinical Psychology and Personality, University of Nijmegen, P.O. Box 9104, 6500 HE, Nijmegen, The Netherlands.

The authors gratefully acknowledge Franny Moene, Pieter Sandijck, and Matthijs Rümke for their support at the General Psychiatric Hospital “De Grote Rivieren”' in Dordrecht. Also many thanks to Dr L.M. Parsons for the hand and foot pictures and his critical comments; to Dr P. Dominey for his thoughtful comments at the initial stage of the study; and to Hubert Voogd for computer support. 
The major feature of conversion disorder is the presence of symptoms affecting voluntary motor or sensory function that suggests neurological disorder (American Psychiatric Association, 1994). Typical of conversion disorder are inconsistencies in the incidence or severity of the sensory and/or motor symptoms. An impairment that appears consistently present, may, under particular circumstances, occur less often or may even be absent. One of the patients we examined, for example, could not move her legs when she was explicitly trying to. Occasionally, when dressing herself, however, she did show some movement of the legs. Inconsistencies such as these are commonly observed in conversion disorder and in his cognitive theory of memory and dissociation Kihlstrom (1992a, b) explains them as follows. Under conditions of overwhelming stress explicit (conscious, intentional) information processing in conversion disorder becomes dissociated from implicit (unconscious, automatic) functioning. In the case of conversion paralysis, this results in impaired intentional motor functioning, whereas automatic motor functioning remains intact. The results of two psychophysiological case studies suggest that in conversion paralysis a mechanism is active that inhibits information processing to occur at levels for awareness (for a review see Sierra \& Berrios, 1999). An event-related potential (ERP) study showed an altered P300 (potential associated with awareness) in a patient with sensory and motor conversion symptoms of the right arm (Lorenz, Kunze, \& Bromm, 1998), whereas the early sensory components of the event-related response were normal. Furthermore, Marshall, Halligan, Fink, Wade, and Frackowiak (1997) recorded brain activity using positron emission tomography (PET) when a patient with a conversion paralysis of the leg prepared and attempted to move the affected leg. There were no abnormalities in brain activation during the preparation phase of the intended movement of the paralysed (left) leg. However, when the patient attempted to move her left leg there was no activation of the right primary motor cortex. Furthermore, the orbitofrontal cortex and the right anterior cingulate cortex were significantly activated. The authors suggested that these two areas seemed to inhibit prefrontal (intentional) effects on the right primary motor cortex when the patient tried to move her left leg. These studies support the view that in conversion paralysis the linkage between higher level and lower level information processes is impaired, resulting in disturbances that predominantly affect intentional motor functioning.

A dissociation between intentional and automatic motor functioning is not unique to conversion disorders. Several neurological syndromes are characterised by a disturbance of the intentional initiation and control of movement whereas motor functioning is otherwise intact. In such syndromes as Parkinson's disease (Dominey, Decety, Broussolle, Chazot, \& Jeannerod, 1995), motor neglect (see Riddoch \& Humphreys, 1994), and ideomotor apraxia (Sirigu et al., 1995a), moreover, difficulties with the formation of mental images of actions are reported. The literature does not report whether 
patients with pseudo-neurological motor symptoms, as observed in conversion paralysis, have impaired mental motor representations. This information is important to gain insight into the intentional motor functions of patients suffering from conversion paralysis, especially since mental motor representations are considered to play a crucial role in the planning and preparation of movement (Jeannerod, 1997). Mental motor representations can be studied through motor imagination tasks in which people are implicitly cued or explicitly instructed to simulate a movement. Studies using such tasks indeed reveal similarities between motor imagination and real movement. Several investigations have, for example, shown no differences in movement time for the performance of real and imaginary movements (Decety, Jeannerod, \& Prablanc, 1989; Decety \& Michel, 1989; Parsons, 1987; Parsons, 1994). Furthermore, motor imagery has been found to evoke increases in heart and respiration rates proportional to the effort of the real movement (Decety, Jeannerod, Durozard, \& Baveral, 1993; Decety, Jeannerod, Germain, \& Pastene, 1991; Wang \& Morgan, 1992). PET measurements of the cerebral blood flow in healthy participants during experiments investigating mental simulation of movements also showed activation of brain areas overlapping with the areas activated during real movement (Decety et al., 1994; Fox, Pardo, Petersen, \& Raichle, 1987; Lang et al., 1994; Parsons et al., 1995; Porro et al., 1996; Stephan et al., 1995).

The present study was designed to find out whether motor impairment in conversion paralysis is also manifest in mental motor representations. Based on the many similarities between real movement capacities and motor imagery capacities (Dominey et al., 1995; Parsons, 1994; Parsons et al., 1995) we expected to find disturbances in the motor imagery of limbs paralysed due to conversion. Because the conversion disorder is generally considered to be a disorder in which the intentional and automatic functions are dissociated, we studied patients with conversion paralysis in both an explicit motor imagery task, in which motor imagery was explicitly instructed, and an implicit motor imagery task, in which motor imagery was indirectly cued. In patients with neurological motor pathology, such as Parkinson's disease, the reaction time profiles on both the implicit and the explicit motor imagery task were very similar. In both tasks the unilaterally affected patients showed a motor imagery asymmetry with a slowing of the affected arm (Dominey et al., 1995). In line with Kihlstrom's dissociation theory and in line with the psychophysiological case studies supporting the view that disturbances take place in intentional processes in conversion disorder, we hypothesised, however, that explicit motor imagery would be more impaired than implicit motor imagery in patients with conversion paralysis. We expected to find a greater degree of motor imagery asymmetry (with a slowing of the affected arm) on the explicit motor imagery task than on the implicit motor imagery task. 


\section{METHOD}

\section{Participants}

Patients applying for either in- or outpatient treatment at a general psychiatric hospital specialising in the treatment of conversion disorders underwent standard psychological, neurological, and psychiatric examination. The patients included in this study were classified as having conversion disorder according to DSM-IV (American Psychological Association, 1994) and had either full or partial paralysis in one or more limbs. Exclusion criteria were symptoms involving pseudo-neurological insults, tremors, sudden movements, or deteriorated speech, vision, or hearing. The patient sample consisted of six righthanded females with a mean age of 37.7 years $(S D=12.3)$. The duration of complaints ranged from 8 to 52 months with a mean of 33 months $(\mathrm{SD}=18.52)$. All of the patients showed normal intellectual functioning in a psychological interview and neuropsychological tests. They were screened for axis-1 comorbidity using the Structured Interview for DSM-IV Axis I Disorders (First, Gibbon, Spitzer, \& William, 1996). Two patients met the criteria for a major depression in remission and used an antidepressant (paroxetine $20 \mathrm{mg} / \mathrm{day}$ ). One patient met the criteria for generalised anxiety disorder and used oxazepam $(10 \mathrm{mg})$ on an irregular basis, but refrained from taking the drug 10 hours prior to the experiment. Table 1 shows an overview of the affected limbs and the relative severity of the impairment. In the present study a comparison is made between the motor imagery performance of the patients' most severely versus

TABLE 1

Severity ratings of patients' affected arms and legs

\begin{tabular}{lcccc}
\hline Patient & Left arm & Right arm & Left leg & Right leg \\
\hline 1 & 1 & 2 & 1 & 2 \\
2 & 1 & $\mathrm{u}$ & $\mathrm{u}$ & $\mathrm{u}$ \\
3 & $\mathrm{u}$ & 1 & 2 & 1 \\
4 & 1 & $\mathrm{u}$ & 1 & 2 \\
5 & 1 & 2 & 1 & 2 \\
6 & 1 & 1 & 1 & 1 \\
\hline
\end{tabular}

Note: The severity ratings were a forced choice with 1 indicating the most severely impaired and 2 indicating the least severely impaired limb. Arms and legs were rated separately. When only one arm or leg was impaired, the rating of 1 was assigned to the affected limb and no rating was assigned to the unaffected limb (u). When both arms or both legs of a patient were equally impaired, the rating of 1 was assigned to both. Note that a difference in the scores for one patient may indicate a large difference between the impaired limbs, whereas the same difference in scores for another patient may indicate a small difference. 
the patients' least severely or nonaffected limbs. To control for "normal", performance differences between limbs due to possible nonspecific factors, such as hand dominance, a nonaffected control group was also studied. The control participants were six healthy right-handed, age-matched females with a mean age of $31.3(\mathrm{SD}=5.1)$. The control participants were recruited via acquaintances and colleagues of the experimenter. All participants provided their informed consent and received the Dutch equivalent of $\$ 10$ for their participation.

\section{Procedure}

Prior to the experiment, the handedness of the participants was assessed. To assess the relative severity of the patient's impairment, the patient was asked to indicate whether one arm or leg was more severely impaired than the other by assigning a rating of " 1 "' to the most severely impaired arm or leg and ' 2 ', to the least severely impaired arm or leg (see Table 1). The attending physiotherapist also made an independent severity assessment for each patient and confirmed the patients' own ratings. The identification of the best and the worst functioning arm and leg in the control subjects was based on the mean reaction times on the motor imagery tasks (Task 3 and 4).

The participants were first familiarised with motor imagery instructions and trained for motor imagery using two imagery questionnaires that require the performance of mental movements. Subsequently, they performed six computer tasks with a total duration of 1.5 to 2 hours (including breaks). The third and fifth tasks were always followed by a 5-minute break. Extra breaks (between tasks) were also allowed when requested. Four of the six tasks formed the basis of the present study and were presented in the following order: (1) a reaction time task, (2) a letter rotation task, (3) an implicit motor rotation task, and (4) an explicit motor rotation task. The other two tasks were used for other research purposes and will be reported on elsewhere.

\section{Materials}

\section{Handedness questionnaire}

Hand preference was assessed using the Annett's Handedness Questionnaire (Annett, 1970). The questionnaire was scored using the method described by Lishman and McMeekan (1976).

\section{Imagery Questionnaires}

Following the motor imagery training procedure by Dominey et al. (1995), all participants were trained for motor imagery by completing two questionnaires that require the performance of mental movements. The Sheehan Imagery Questionnaire (Sheehan, 1967) measures the ability to imagine a variety of perceptual and motor experiences. The Movement Imagery Questionnaire (Hall, 
Pongrac, \& Buckholz, 1985) measures the quality of imagery for 18 items involving the real performance of a movement followed by the mental performance of the same movement. If a patient was unable to perform the real movement, the experimenter demonstrated the movement prior to imagination. Only those items involving an isolated arm or leg movement were used (items 1, $2,5,6,10,11,14,15)$.

\section{Computer Tasks}

The computer tasks were programmed on a Macintosh computer (LC475). Each task was preceded by computerised instructions, an example with feedback, and a practice phase in which all task stimuli were shown once. The stimuli appeared in the centre of the screen and remained visible until the participant responded using a voice key attached to a headset. Reaction times (RTs) were recorded using an electronic timer with an accuracy of approximately $1 \mathrm{~ms}$. The experimenter recorded each answer and started the next stimulus presentation. The stimulus presentations were preceded by the presentation of a fixation point, lasting $300 \mathrm{~ms}$, in the centre of the screen followed by a blank screen, which lasted $200 \mathrm{~ms}$. The participants were seated in a chair with lengthened arm rests. As the actual position of one's body influences the time needed to mentally perform a movement (Parsons, 1994), for the duration of the experiment the participants were asked to position their hands on the arm rests palms down and pointing forward, and to place their feet flatly on an adjustable footstool, toes pointing forward. The distance between the participants' eyes and the centre of the screen was $75 \mathrm{~cm}$.

Task 1: Reaction time task. In order to examine the possibility of a general reaction time (RT) difference between the two groups a simple RT task was administered. Two stimuli, a circle (diameter $0.55 \mathrm{~cm}$ ) and a square (sides 1.10 $\mathrm{cm}$ ), were each presented 24 times in random order. The participants were instructed to identify the presented stimulus as quickly and as accurately as possible by saying "circle" or "square", out loud.

Task 2: Letter rotation. This mental letter rotation task provides an index of a general imagery capacity, whereas mental rotation of hands and feet (see Tasks 3 and 4) relies on more specific mechanisms of motor imagery (Dominey et al., 1995; Parsons, 1987, 1994). The letter rotation task implicitly evokes mental letter rotation and is used as a control task for the implicit motor rotation task (Task 3$)$. The stimuli were the capital letters $F$ or $\mathrm{R}(1.5 \mathrm{~cm}$ in height) written in Times (60 points). The letters were presented in either mirror-reversed or normal orientation at 8 angles between $0^{\circ}$ and $315^{\circ}$ with $45^{\circ}$ increments. The test phase consisted of two blocks with 32 randomly presented trials each (2 letters $\times 2$ versions $\times 8$ angles). The participants were instructed to determine as quickly 
and as accurately as possible whether a letter was presented in normal or mirror orientation by loudly and clearly stating "normal" or "mirror".

Task 3: Implicit motor rotation. This decision task involves the implicit mental rotation of hands and feet (Parsons, 1994; Parsons et al., 1995; Parsons, Gabrieli, Phelps, \& Gazzaniga, 1998). The stimuli were Parsons' line drawing pictures of hands $(6.5 \mathrm{~cm}$ high and $5 \mathrm{~cm}$ wide $)$ and feet $(5.5 \mathrm{~cm}$ high and $2.4 \mathrm{~cm}$ wide). Both "palm" and "back" versions of the left and right hands and "top" and "sole" versions of left and right feet were presented. All pictures were presented at 8 rotation angles ranging from $0^{\circ}$ to $315^{\circ}$ with $45^{\circ}$ increments. All stimuli were tested in a pilot study with eight healthy participants. Due to physical constraints, some of the positions for the feet could not be reached while seated. For this reason, the $135^{\circ}, 180^{\circ}$, and $225^{\circ}$ pictures of both the left and right foot, tops and soles were omitted from the test. The participants were instructed to decide as quickly and as accurately as possible whether the presented hand or foot was a left or right one by loudly and clearly saying "left", or "right". In the test phase all possible presentations for the hands $(2$ hands $\times$ 2 versions $\times 8$ angles $=32)$ and feet $(2$ feet $\times 2$ versions $\times 5$ angles $=20)$ were presented twice. The hand and foot stimuli were randomly assigned to blocks of 8 hands or 5 feet, respectively. The blocks were presented in a random order and the type of stimulus (hands or feet) was indicated on the screen prior to the presentation of a block.

Task 4: Explicit motor rotation. In order to investigate the relation between implicit and explicit motor imagery in conversion disorders, the participants were presented with the hand and foot stimuli of Task 3 but now with the explicit instruction to mentally rotate their hands or feet in a natural and efficient manner without actually moving to the positions presented on the screen and to say "yes" when they had reached the target position. The procedure for stimulus presentation was the same as in Task 3, except that the stimuli were randomly presented in blocks of left hands, right hands, left feet and right feet. The blocks were presented in a random order and the type of stimulus (left or right, hands or feet) was indicated on the screen prior to the presentation of a block. The experimenter watched to see that no movements were actually made. In addition, videotapes were made.

\section{Data analyses}

In the mental rotation tasks (Tasks 2-4) not the direction but the length of rotation was of interest for the present study. Because the $45^{\circ}$ and $315^{\circ}, 90^{\circ}$ and $270^{\circ}$, and $135^{\circ}$ and $225^{\circ}$ rotations equally differ from the $0^{\circ}$ position, the data were collapsed for each pair. This resulted in 5 orientation differences (OD) of $0^{\circ}, 45^{\circ}, 90^{\circ}, 135^{\circ}$, and $180^{\circ}$. On each task, RTs greater than two standard deviations (SD) from the individual mean RT per OD (and per limb in Tasks 3 
and 4) were attributed to distraction or a loss of attention and therefore excluded from the analyses. For each participant, the mean RT of the correct responses was then calculated per OD for every task.

\section{Task 1: Reaction time task}

The possibility of a general group difference between patients and controls on the reaction time task (1) was tested using a $t$-test for independent samples.

\section{Task 2: Letter rotation task}

In mental rotation tasks the RT is found to linearly increase as a function of the angle of rotation (Shepard \& Cooper, 1982; Shepard \& Metzler, 1971). As a method for data reduction individual regression analyses for RT by OD were conducted resulting in two variables per person. The slope of the regression function is the relative RT increase per rotation degree which provides an estimate of the rate at which the mental manipulation can be carried out. The intercept is the estimated RT without rotation load (Shepard \& Metzler, 1971). To assess a possible group difference in regression intercept, the individual intercepts were put to a two-tailed multivariate analysis of variance (MANOVA) with Group (Patients, Controls) as between-subject factor. To assess a possible group difference in the regression slope the same was done with the individual slopes as dependent variable.

\section{Task 3 and 4: Implicit and explicit motor rotation tasks}

To test for asymmetry in motor imagery functioning due to the impairment of the limbs, for each patient the best and worst functioning arm and the best and worst functioning leg were identified (see Table 1). This was also done for the control group on the basis of the mean RTs per limb on Tasks 3 and 4. Individual regression analyses were conducted for each limb. Because the number of angles of rotations used differed for hands and feet, the data for hands and feet were analysed separately.

To test our hypothesis that mental rotation of the (most severely) affected arm is slower in patients, the individual intercepts associated with the worst and the best functioning arm were put to a one-tailed MANOVA with Group (Patients, Controls) as between-subject factor and Arm (Best, Worst) as within-subject factor. The same analysis was conducted for legs with factor Leg (Best, Worst) as within-subject factor.

To test our hypothesis that the slowing associated with an increase in angle of rotation is larger for motor imagery of the (most severely) affected arm in patients, the individual regression slopes for both arms were put to a one-tailed MANOVA with Group as between-subject factor and Arm as within-subject factor. The same analysis was conducted for the legs. 


\section{RESULTS}

Reaction time task (1)

Significant group differences were observed on the reaction time task. The result of a $t$-test for independent samples showed the mean RT (ms) of the patients (723, $S D=183)$ to be significantly $(p<.05)$ slower than the mean RT of the controls $(484, S D=59)$.

\section{Letter rotation task (2)}

To evaluate how much variance could be explained by the linear relation between angle and RT we computed multiple correlations $\left(R^{2}\right)$ of the individual regression lines using the mean RT per OD. The mean $R^{2}$ of the individual regression lines for the letter rotation RTs was .44 for controls and .54 for patients.

$R T$-intercept. A MANOVA with the intercept of the individual regression lines as dependent variable and Group (Patients, Controls) as between-subject factor showed that the mean intercept of the patients $(873 \mathrm{~ms})$ did not significantly differ from that of the controls $(743 \mathrm{~ms}), F(1,10)=2.17, p=.17$.

Slope. A MANOVA with slope as dependent variable and Group as between-subject factor showed that the slope of the patients' regression line (3.93) was significantly larger than the slope of the controls' regression line (1.36), $F(1,10)=6.95, p<.05$.

These results indicate that, although the estimated RT for $0^{\circ}$ letter rotation (intercept) was equal for both groups, patients rotated more slowly than controls.

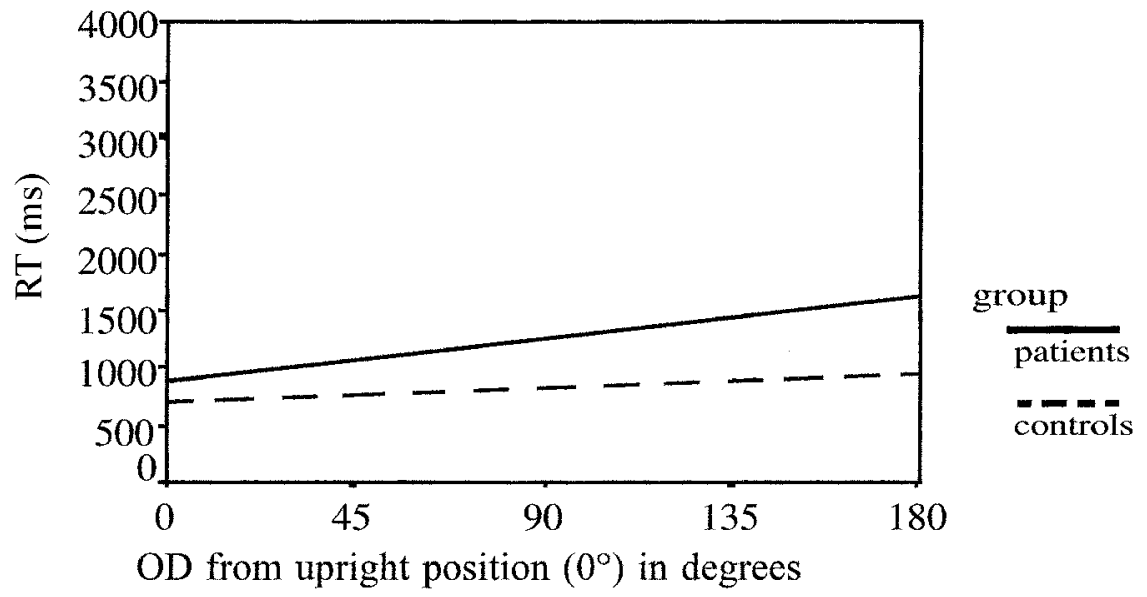

Figure 1. Regression lines of mental letter rotations of control subjects $(n=6)$ and conversion patients $(n=6)$; OD $=$ orientation difference. 


\section{Implicit motor rotation task (3)}

\section{Implicit hand rotation}

The mean $R^{2}$ for the controls' regression lines was .69 for the best and .51 for the worst functioning arm. For patients the mean $R^{2}$ s were .69 (best functioning arm) and .78 (worst functioning arm).

RT-intercept. A two-way MANOVA with Arm (Best, Worst) as withinsubject factor and Group (Patients, Controls) as between-subject factor showed no main effect for Arm (Best: 1103, Worst: $1104 \mathrm{~ms}), F(1,20)=.00, p=.49$. A significant main effect for Group was found: The patients' intercept (1284 ms) was significantly larger than the intercept of the controls $(925 \mathrm{~ms}), F(1,20)=$ $5.81, p<.05$. There was no significant interaction between Arm and Group, $F(1,20)=.24, p=.31$.

Slope. A two-way MANOVA with Arm as within-subject factor and Group as between-subject factor showed a marginal effect for Arm (Best: 3.68, Worst: $6.51), F(1,20)=2.75, p=.06$. A significant main effect for Group was found: The slope was significantly larger for patients (7.79) than for controls (2.40), $F(1,20)=9.93, p<.01$. The interaction between Arm and Group was not significant, but we did observe a trend that indicates that patients, more than controls, are slowed when mentally rotating the worst functioning arm, $F(1,20)$ $=2.45, p=.07$ (see Figure 2).

These results indicate that on the implicit hand rotation task patients were significantly slower on the $0^{\circ}$ hand rotation than controls. Furthermore, the slope was significantly larger for patients than for controls but only showed a (nonsignificant) slowing for patients when they were mentally moving the most severely affected arm.

\section{Implicit foot rotation}

The mean $R^{2}$ for the controls' regression lines was .62 for the best and .60 for the worst functioning leg. For patients the mean $R^{2}$ s were .79 (best functioning leg) and .58 (worst functioning leg).

RT-intercept. A two-way MANOVA with Leg (best, worst functioning) as within-subject factor and Group (Patients, Controls) as between-subject factor showed no main effect for Leg (Best: 1011, Worst: $1024 \mathrm{~ms}), F(1,20)=.01, p=$ .46 or Group (Patients: 1178 , Controls: $857 \mathrm{~ms}$ ) on the intercept, $F(1,20)=5.69$, $p<.05$. There was also no significant interaction between Leg and Group, $F(1,20)=.08, p=.40$.

Slope. A two-way MANOVA with Leg as within-subject factor and Group as between-subjects factor showed no main effect for Leg (Best: 5.68, Worst: 


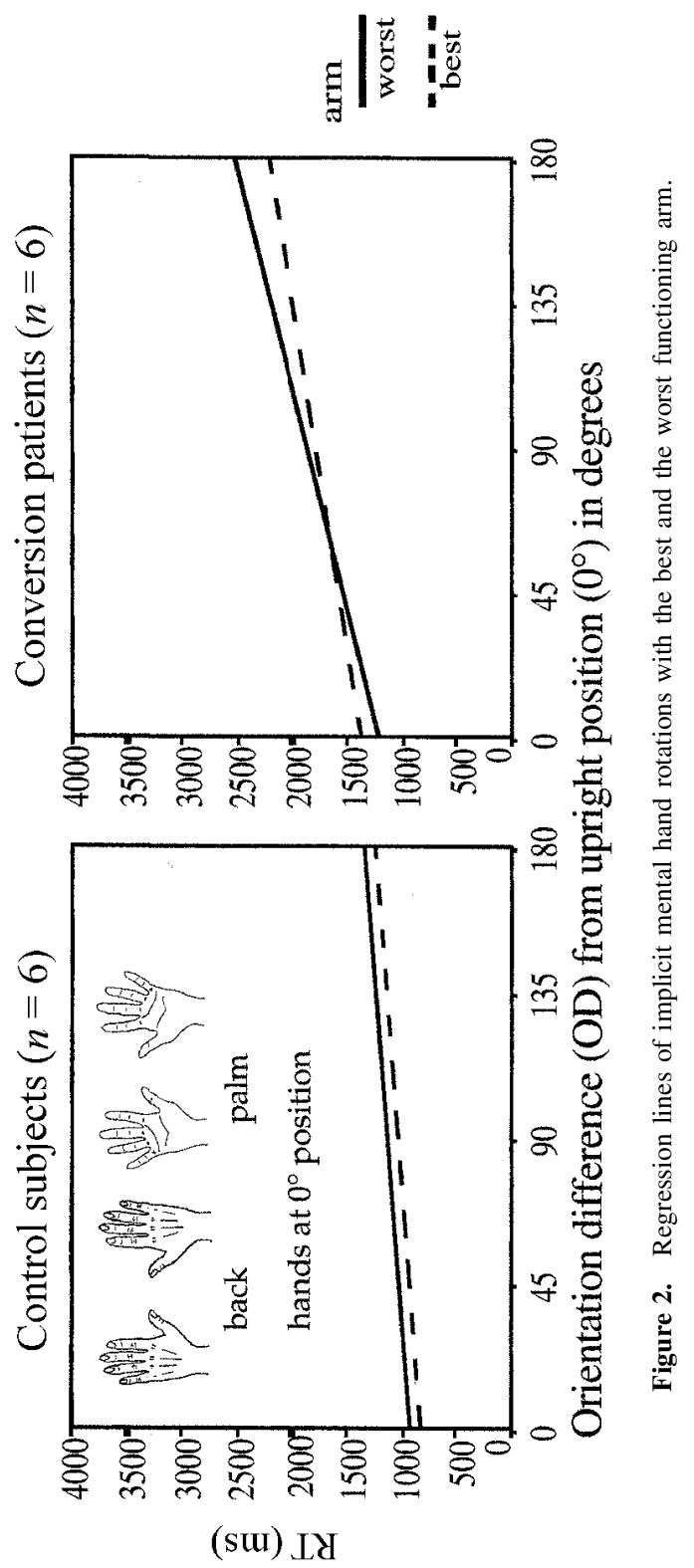


4.99), $F(1,19)=.05, p=.41$. A significant main effect for Group was found: The slope was significantly larger for patients (8.85) than for controls (2.15), $F(1,19)=11.37, p<.01$. There was no significant interaction between Leg and Group, $F(1,19)=.27, p=.31$.

The implicit foot rotations showed similar results to the implicit hand rotations: the slopes and the intercepts were significantly larger for patients than for controls and both the intercept and the slope showed no significant differentiation for Leg (see Figure 3).

\section{Explicit motor rotation task (4)}

\section{Explicit hand rotations}

The mean $\mathrm{R}^{2}$ for the controls' regression lines was .72 for the best and .83 for the worst functioning arm. For patients the mean $\mathrm{R}^{2} \mathrm{~s}$ were .62 (best functioning arm) and .68 (worst functioning arm).

In a total of $8.9 \%$ (34) of the trials patients reported an inability to imagine their hand rotating to the target position versus $0.5 \%$ (2) reported by the controls. One patient was completely unable to form a mental image of her affected arm. Because of the absence of RTs for the "worst functioning arm" she had to be excluded from the analyses.

RT-Intercept. A two-way MANOVA with Arm as within-subject factor and Group as between-subject factor showed no main effect for Arm (Best: 1337, Worst: $1568 \mathrm{~ms}), F(1,18)=1.06, p=.16$. A significant main effect for Group was found: the mean intercept for patients $(1776 \mathrm{~ms})$ was significantly larger than that for controls $(1166 \mathrm{~ms}), F(1,18)=8.66, p<.01$. There was no significant interaction between Arm and Group, $F(1,18)=.26, p=.31$.

Slope. A two-way MANOVA with Arm as within-subject factor and Group as between-subject factor was conducted. There was a main effect for Arm, $F(1,18)=3.01, p<.05$. The slope of the regression line for the worst arm (6.82) was larger than the slope for the best arm (4.05). There was also a main effect for Group, $F(1,18)=3.11, p<.05$. The slope of the regression line for patients (6.9) was larger than the slope for controls (4.02). Finally, there was an interaction between Group and Arm, $F(1,18)=3.04, p<.05$.

These results indicate that on the explicit hand rotation task the intercept was larger for patients than for controls. Furthermore, the slope of the worst functioning hand was significantly larger than the slope for the best functioning arm and this effect was significantly larger in patients than in controls (see Figure 4). Finally, an inability to mentally move the arms was more frequently reported by patients than by controls. 

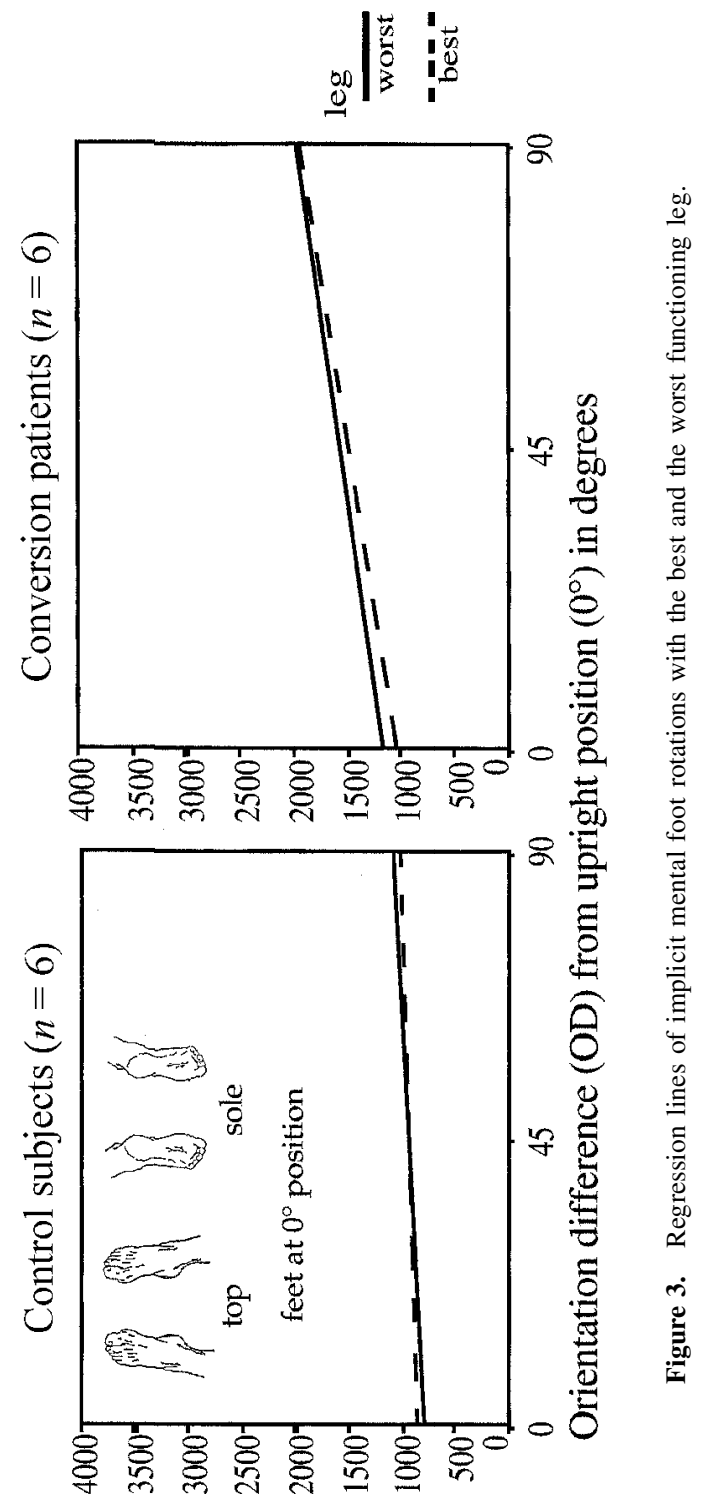

(sui) LY 


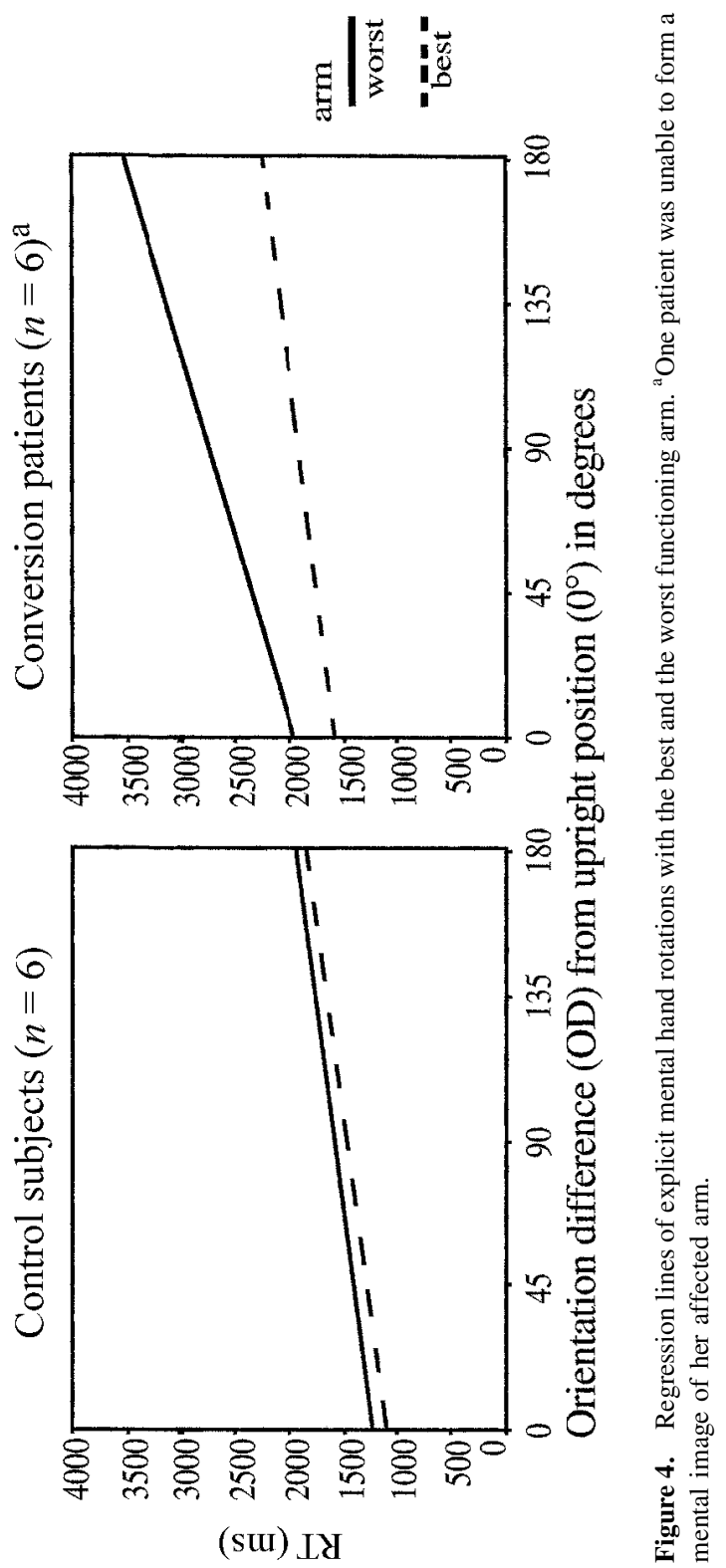




\section{Explicit foot rotation}

Interestingly, patients were unable to imagine their foot moving into the target position in $51.3 \%$ (121) of the trials, whereas this was $0 \%$ for the controls. This relatively large absence of patients' RTs in the foot rotation task and the fact that the data for the larger rotations were predominantly absent rendered the data unreliable and not suitable for statistical analysis.

\section{DISCUSSION}

The objective of the present study was to find out whether patients with conversion paralysis show impairments in mental motor representations. Based on the many similarities between real movement and motor imagery in subjects with normal (Parsons, 1994) and pathological movement constraints (Dominey et al., 1995; Parsons et al., 1998; Sirigu et al., 1995b) we hypothesised first that we would find impaired motor imagery in patients with paralysed limbs as a result of conversion. Because patients with conversion disorder typically show deficits of intentional (willed) movements it was secondly hypothesised that this impairment would be larger for explicit motor imagery than for implicit motor imagery.

Three findings resulted from the study. First, the results showed that patients were significantly slower than controls on all RT tasks, including a relatively easy one. As will be argued later, these results suggest a general psychomotor slowing of patients with conversion paralyses.

Second, patients with conversion paralyses showed a larger RT increase per degree of rotation (slope of regression line) than controls on the implicit mental hand and foot rotation task. This finding, indicating that patients mentally rotated their limbs more slowly than controls, was unexpected. However, a similar effect was found in the mental letter rotation task. Patients showed a larger RT increase per degree of rotation than controls. These results suggest that mental rotation in general is slowed in patients with conversion paralysis compared to controls. In addition, patients were slower than controls on the $0^{\circ}$ rotations of the implicit motor rotation task and a similar trend was observed in the letter rotation task. No significant effects for limb (best vs. worst functioning) were found on the implicit hand and foot imagery task, although the implicit hand rotations did show a (nonsignificant) trend towards slowing in the patients' performance with the most severely affected (worst functioning) arm.

Thirdly, the psychomotor slowing observed in patients with conversion paralysis most strongly manifested itself during the explicit motor rotation task. Patients were significantly slower than controls when explicitly instructed to mentally move their limbs. Furthermore, patients' explicit motor imagery of the most severely affected arm as a function of movement size was slower than the explicit motor imagery of the least severely or nonaffected arm. One patient was 
unable to form a mental image of her most severely affected arm. Such a complete inability to form mental motor representations was also reported by patients for explicitly evoked mental rotations of the feet. In $51.3 \%$ of the trials patients reported to be unable to mentally move their legs, in most cases the most severely affected limb. The predominance of this inability to mentally rotate the legs is probably due to the fact that, in comparison to the upper limbs, the lower limbs of the conversion patients were more severely impaired.

Our findings support the hypothesis that explicitly instructed (intentional or willed) generation of mental movement with affected limbs is most strongly disturbed in conversion paralysis. Whereas neurological motor pathology such as in Parkinson's disease, is associated with an asymmetry (a larger slowing of the affected arm) in both implicitly and explicitly instructed motor imagery, conversion paralysis is associated with such asymmetry only when motor imagery is explicitly instructed. Thus, patients with conversion paralysis were predominantly impaired when their motor imagery was intentionally generated. These findings are in agreement with the findings of Marshall et al.'s study (1997), showing abnormalities in brain activity only when a patient deliberately attempted to move. The findings are also in agreement with previous case studies, suggesting that conversion disorder is associated with an impairment of higher cognitive processes, such as those requiring intentionality and/or awareness. The results of a brain mapping study of Tiihonen, Kuikka, Viinamaki, Lehtonen, and Partanen (1995), for example, suggested the involvement of frontal inhibitory areas in conversion paresthesia. And the findings of two ERP studies suggested an impairment at a high level of cognitive processing in two cases of sensory conversion disorder (Fekuda et al., 1996; Lorenz et al., 1998).

The results also suggest that the impaired motor processing in conversion paralysis already manifests itself in movement preparation. As argued in the introduction, motor imagery is important for the planning and preparation of movement (Jeannerod, 1997). Just like real movement, motor imagery requires an interaction between current somatosensory information and motor programmes that are stored in memory. Findings from a PET study (Parsons \& Fox, 1998), for example, suggested the performance of the implicit hand rotation task to depend on lateralised sensorimotor and somatosensory representations. This interaction between the current somatosensory information and stored motor programmes is likely to fail when the patient with conversion paralysis intentionally generates motor imagery.

We were surprised to find that conversion patients were also slowed on the two RT-tasks involving object identification. However, similar to implicit and explicit mental motor rotation tasks, these tasks also require the intentional control of movement, as they demand subjects to produce a verbal response on the identification of a visual stimulus. A possible explanation for the general slowing as reflected by the larger RTs on all performed tasks is a disturbance of 
the intentional control as part of the whole system. This explanation is in accordance with dissociation theories of conversion disorder that state that under severe stress the human information processing system as a whole breaks down into more or less separately functioning subsystems (Hilgard, 1977; Kihlstrom, 1992b; Nehmia, 1991; Schacter \& Kihlstrom, 1989; Spiegel, 1990). This disintegration or compartmentalisation allows subsystems to continue functioning without explicit initiation or awareness. The intentional control over the subsystems is, however, weakened and the integrity of the information processing system, which normally shows a fluent cooperation between subsystems, is disturbed. Under these disturbed conditions, the output of the system as a whole is likely to be qualitatively worse. Compartmentalisation might, therefore, explain the general slowing of the higher cognitive, intentional processes that require a high degree of cooperation between different subsystems. Likewise, one might expect that in patients with conversion paralysis the motor processes that are not specifically affected or "nonsymptomatic" (e.g., speech) will not display impaired movement execution but rather an impairment of the intentional initiation of the movement. An objection to the proposed compartmentalisation hypothesis is that a possible influence of depressive symptomatology and medication cannot be excluded. The observed general slowing may be attributable to these two factors. However, they cannot explain the differential effects of limb and explicitly instructed motor imagery. Furthermore, in a recent study, in which we compared motor initiation (RT) and motor execution (speech time) on the four RT tasks of the present study, we found that the differential effects for limb (best vs. worst functioning) and instruction (implicit vs. explicit) were specific for RT and not for speech time. These results suggest that the initiation rather than the execution of movement is disturbed. The results therefore support compartmentalisation hypothesis in conversion paralysis (Roelofs, Van Galen, Keijsers, \& Hoogduin, 2000).

A limitation of the present study is that the patients with conversion paralysis who took part in the experiment were not systematically unilaterally impaired. Comparison of the best with the worst functioning arm did show an effect at the .05 significance criteria in the explicit hand rotation task and a nonsignificant trend in the implicit hand rotation task. A replication of the present study with patients having unilateral conversion paralysis may more clearly demonstrate these effects. Furthermore, the control group of nonmotor affected subjects allows us to conclude that the differential effect for arm is complaint-specific and not attributable to a possible "normal" performance difference between left and right limbs, related to nonspecific factors such as hand dominance. The additional finding of a general slowing of the conversion disorder patients, however, demands further research using a control group of patients known to have a general (task unspecific) slowing (e.g., patients with depressive disorder; White, Myerson, \& Hale, 1997). Such a control group would allow the proposed compartmentalisation hypothesis to be further tested. 
A final remark concerns two therapeutic implications of the finding of impaired explicit motor imagery in conversion paralysis. First, the disturbances in mental motor representations may offer a starting point for therapeutic interventions. Motor imagery training has been shown to improve several aspects of motor performance, such as movement speed (Pascual-Leone et al., 1995 ) and muscular strength (Yue \& Cole, 1992), in healthy subjects and may be a promising strategy in the treatment of conversion patients. Second, reactivation of the motor system is an important first step in therapy (Moene, Hoogduin, \& Van Dyck, 1998). To achieve this, the use of indirect techniques that bypass the disturbed intentional processes may be more fruitful than the use of direct strategies.

Manuscript received 7 January 2000

Revised manuscript received 13 July 2000

\section{REFERENCES}

American Psychiatric Association. (1994). Diagnostic and statistical manual of mental disorders (4th ed.). Washington, DC: Author.

Annett, M. (1970). Classification of hand preference by association analysis. British Journal of Psychiatry, 61, 303-321.

Decety, J., Jeannerod, M., Bettinardi, V., Tardary, B., Woods, R., Mazziotta, J.C., \& Fazio, F. (1994). Mapping motor representations with PET. Nature, 371, 600-602.

Decety, J., Jeannerod, M., Durozard, D., \& Baveral, G. (1993). Central activation of autonomic effector during mental simulations of motor actions in man. Journal of Physiology, 461, 540-563.

Decety, J., Jeannerod, M., Germain, M., \& Pastene, J. (1991). Vegetative response during imagined movement is proportional to mental effort. Behavioural Brain Research, 34, 35-42.

Decety, J., Jeannerod, M., \& Prablanc, C. (1989). The timing of mentally represented actions. Behavioural Brain Research, 34, 35-42.

Decety, J., \& Michel, F. (1989). Comparative analysis of actual and mental movement times in two graphic tasks. Brain and Cognition, 11, 87-97.

Dominey, P., Decety, J., Broussolle, E., Chazot, G., \& Jeannerod, M. (1995). Motor imagery of lateralized sequential task is asymmetrically slowed in hemi-Parkinson's patients. Neuropsychologia, 33, 727-741.

Fekuda, M., Hata, A., Niwa, S., Hiramatsu, K., Yokokoij, M., Hayashida, S., Itok, K., Nakagome, K., \& Iwanami, A. (1996). Event-related potential correlates of functional hearing loss: reduced P3 amplitude preserved N1 and N2 components in a unilateral case. Neuropsychiatry and Clinical Neuroscience, 50, 85-87.

First, M.B., Gibbon, M., Spitzer, R.L., \& William, J.B.W. (1996). Structured clinical interview for DSM-IV axis I disorders, version 2.0. New York: Biometrics Research.

Fox, P.T., Pardo, J.V., Petersen, S.E., \& Raichle, M.E. (1987). Supplementary motor and premotor responses to actual and imagined hand movements with PET. Neuroscientific Abstracts, 13, 1433.

Hall, C., Pongrac, J., \& Buckholz, E. (1985). The measurement of imagery ability. Human Movement Science, 4, 107-118.

Hilgard, E.R. (1977). Divided consciousness: Multiple controls in human thought and action. New York: Wiley. 
Jeannerod, M. (1997). The cognitive neuroscience of action. Oxford: Blackwell.

Kihlstrom, J.F. (1992a). Dissociation and dissociations: A comment on consciousnes s and cognition. Consciousness and Cognition, 1, 47-53.

Kihlstrom, J.F. (1992b). Dissociative and conversion disorders. In D.J. Stein \& J.E. Young (Eds.), Cognitive science and clinical disorders. San Diego, CA: Academic Press.

Lang, W., Petit, L., Höllinger, P., Pietrzyk, U., Tzourio, N., Mazoyer, B., \& Berthoz, A. (1994). A positron emission tomography study of oculomotor imagery. Neuro Report, 5, 921-924.

Lishman, W.A., \& McMeekan, E.R.L. (1976). Hand preference patterns in psychiatric patients. British Journal of Psychiatry, 129, 158-166.

Lorenz, J., Kunze, K., \& Bromm, B. (1998). Differentiation of conversive sensory loss and malingering by P300 in a modified oddball task. Neuro Report, 9, 187-191.

Marshall, J.C., Halligan, P.W., Fink, G.R., Wade, D.T., \& Frackowiak, R.S.J. (1997). The functional anatomy of a hysterical paralysis. Cognition, 64, B1-B8.

Moene, F.C., Hoogduin, C.A.L., \& Van Dyck, R. (1998). The inpatients treatment of patients suffering from (motor) conversion symptoms: A description of eight cases. International Journal of Clinical and Experimental Hypnosis, XLVI, 171-190.

Nehmia, J.C. (1991). Dissociation, conversion and somatization. In A. Tasman \& S.M. Goldfinger (Eds.), American Psychiatric Press Review (Vol. 10). Washington, DC: American Psychiatric Press.

Parsons, L.M. (1987). Imagined spatial transformations of one's hands and feet. Cognitive Psychology, 19, 178-241.

Parsons, L.M. (1994). Temporal and kinematic properties of motor behavior reflected in mentally simulated action. Journal of Experimental Psychology, Human Perception and Performance, 20, 709-730.

Parsons, L.M., \& Fox, P.T. (1998). The neural basis of implicit movements used in recognising hand shape. Cognitive Neuropsychology, 15, 583-615.

Parsons, L.M., Fox, P.T., Downs, J.H., Glass, T., Hirsch, T.B., Martin, C.C., Jerabek, P.A., \& Lancaster, J.L. (1995). Use of implicit motor imagery for visual shape discrimination as revealed by PET. Nature, 375, 54-58.

Parsons, L.M., Gabrieli, J.D.E., Phelps, E.A., \& Gazzaniga, M.S. (1998). Cerebrally lateralized mental representations of hand shape and movement. Journal of Neuroscience, 18, 6539-6548.

Pascual-Leone, A., Dang, N., Cohen, L.G., Brasil-Neto, J., Cammarota, A., \& Hallett, M. (1995). Modulation of motor responses evolved by transcranial magnetic stimulation during the acquisition of new fine motor skills. Journal of Neurophysiologica l, 74, 1037-1045.

Porro, C.A., Francescato, M.P., Cettolo, V., Diamond, M.E., Baraldi, P., Zuiani, C., Bazzocchi, M., \& Prampero, P.E. (1996). Primary motor and sensory cortex activation during motor performance: A functional magnetic resonance imagery study. Journal of Neuroscience, 16, 7688-7698.

Riddoch, M.J., \& Humphreys, G.W. (Eds.) (1994). Cognitive neuropsychology and cognitive rehabilitation. Hove, UK: Lawrence Erlbaum Associates Ltd.

Roelofs, K., Van Galen, G.P., Keijsers, G.P.J., \& Hoogduin, C.A.L. (2000). Motor initiation and motor execution in patients with conversion paralysis. Manuscript in preparation.

Schacter, D.L., \& Kihlstrom, J.F. (1989). Functional amnesia. In F. Boller \& G.J (Eds.), Handbook of neuropsychology (Vol. 3). Amsterdam: Elsevier.

Sheehan, P.W. (1967). A shortened form of Bett's questionnaire upon Mental Imagery. Journal of Clinical Psychology, 23, 386-389.

Shepard, R., \& Cooper, L. (1982). Mental images and their transformations. Cambridge, MA: MIT Press.

Shepard, R.N., \& Metzler, J. (1971). Mental rotation of three-dimensional objects. Science, 171, 171-703.

Sierra, M., \& Berrios, G.E. (1999). Towards a neuropsychiatry of conversive hysteria. Cognitive Neuropsychiatry, 4(3), 267-287. 
Sirigu, A., Cohen, L., Duhamel, J.R., Pillon, B., Dubois, B., \& Agid, Y. (1995a). A selective impairment of hand posture for object utilization in apraxia. Cortex, 31, 41-56.

Sirigu, A., Cohen, L., Duhamel, J.R., Pillon, B., Dubois, B., Agid, Y., \& Pierrot-Deseiligny, C. (1995b). Congruent unilateral impairments for real and imagined movements. NeuroReport, 6, 997-1001.

Spiegel, D. (1990). Hypnosis, dissociation and trauma. In J.L. Singer (Ed.), Repression and dissociation: Implications for personality, theory, psychopathology and health. Chicago, IL: University of Chicago Press.

Stephan, K.M., Fink, G.R., Passingham, R.E., Silbersweig, D., Ceballos-Bauman, A.O., Frith, C.D., \& Frackowiak, R.S. (1995). Functional anatomy of the mental representation of upper extremity movements in healthy subjects. Journal of Neurophysiology, 73, 373-386.

Tiihonen, J., Kuikka, J., Viinamaki, H., Lehtonen, J., \& Partanen, J. (1995). Altered cerebral bloodflow during hysterical paresthesia. Biological Psychiatry, 15, 134-135.

Wang, Y., \& Morgan, W.P. (1992). The effects of imagery perspectives on the physiological responses to imagined exercise. Behavioural Brain Research, 52, 167-174.

White, D.A., Myerson, J., \& Hale, S. (1997). How cognitive is psychomotor slowing in depression? Evidence from a meta-analysis. Aging, Neuropsychology, and Cognition, 4, 166-174.

Yue, G., \& Cole, K.J. (1992). Strength increases from the motor program. Comparison of training with maximal voluntary and imagined muscle contractions. Journal of Neurophysiology, 67, $1114-1123$. 